Supporting Information for

\title{
Boosting catalytic lifetime in chemical vapor deposition of carbon nanotubes by rapid thermal pretreatment of alumina-supported metal nanocatalysts
}

Jaegeun Lee ${ }^{1,2 \dagger}$, Golnaz Najaf Tomaraei ${ }^{1 \dagger}$, Moataz Abdulhafez ${ }^{1}$, and Mostafa Bedewy $y^{1,3,4 *}$

${ }^{1}$ Department of Industrial Engineering, University of Pittsburgh, 3700 O’Hara Street, Pittsburgh, PA 15261, USA

${ }^{2}$ Department of Organic Material Science and Engineering, Pusan National University, 2,

Busandaehak-ro 63 beon-gil, Geumjeong-gu, Busan, 46241, Republic of Korea

${ }^{3}$ Department of Chemical and Petroleum Engineering, University of Pittsburgh, 3700 O'Hara

Street, Pittsburgh, PA 15261, USA

${ }^{4}$ Department of Mechanical Engineering and Materials Science, University of Pittsburgh, 3700

O’Hara Street, Pittsburgh, PA 15261, USA

†These authors contributed equally to this work.

*author to whom correspondence should be addressed.

E-mail address: mbedewy@pitt.edu (M. Bedewy) 


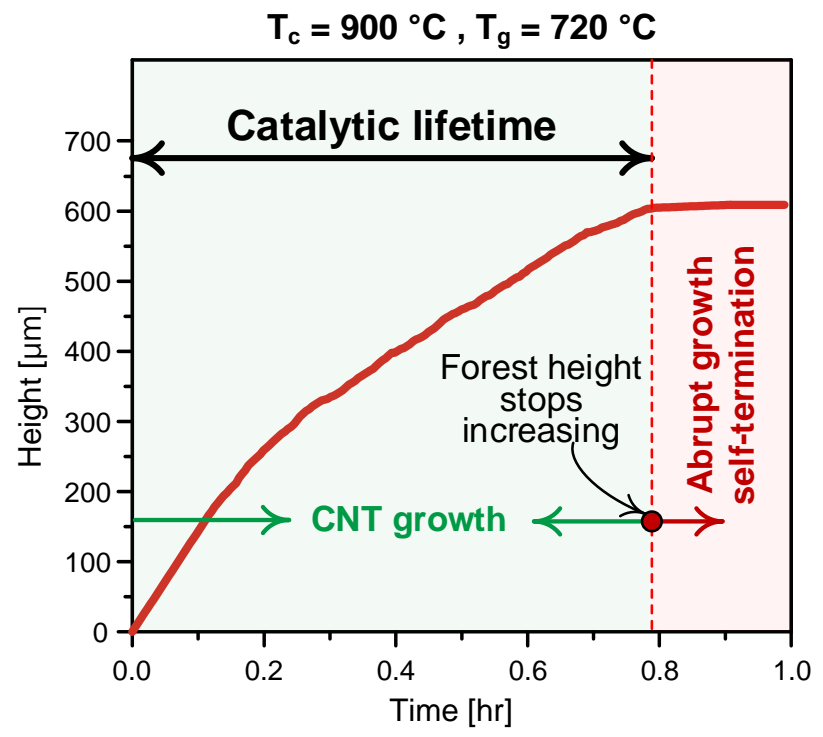

Fig. S1. A representative growth kinetics curve for a CNT forest, which illustrates the method of obtaining the "catalytic lifetime" in this work. Here, catalytic lifetime refers to the time spanning the start of growth and the point at which forest height suddenly stops increasing due to abrupt growth self-termination. 

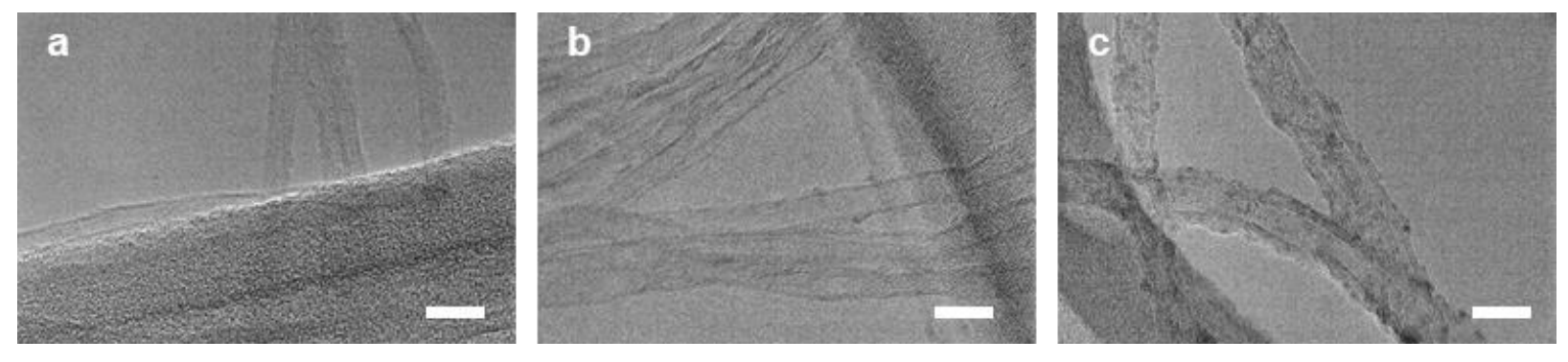

Fig. S2. Representative TEM images of CNTs grown at various combinations of $T_{\mathrm{g}}$ and $T_{\mathrm{c}}$ with other conditions fixed: (a) $T_{\mathrm{g}}=700^{\circ} \mathrm{C}$ and $T_{\mathrm{c}}=600{ }^{\circ} \mathrm{C}$, (b) $T_{\mathrm{g}}=700{ }^{\circ} \mathrm{C}$ and $T_{\mathrm{c}}=700{ }^{\circ} \mathrm{C}$, (a) $T_{\mathrm{g}}=$ $900{ }^{\circ} \mathrm{C}$ and $T_{\mathrm{c}}=700{ }^{\circ} \mathrm{C}$. All scale bars are $20 \mathrm{~nm}$. 

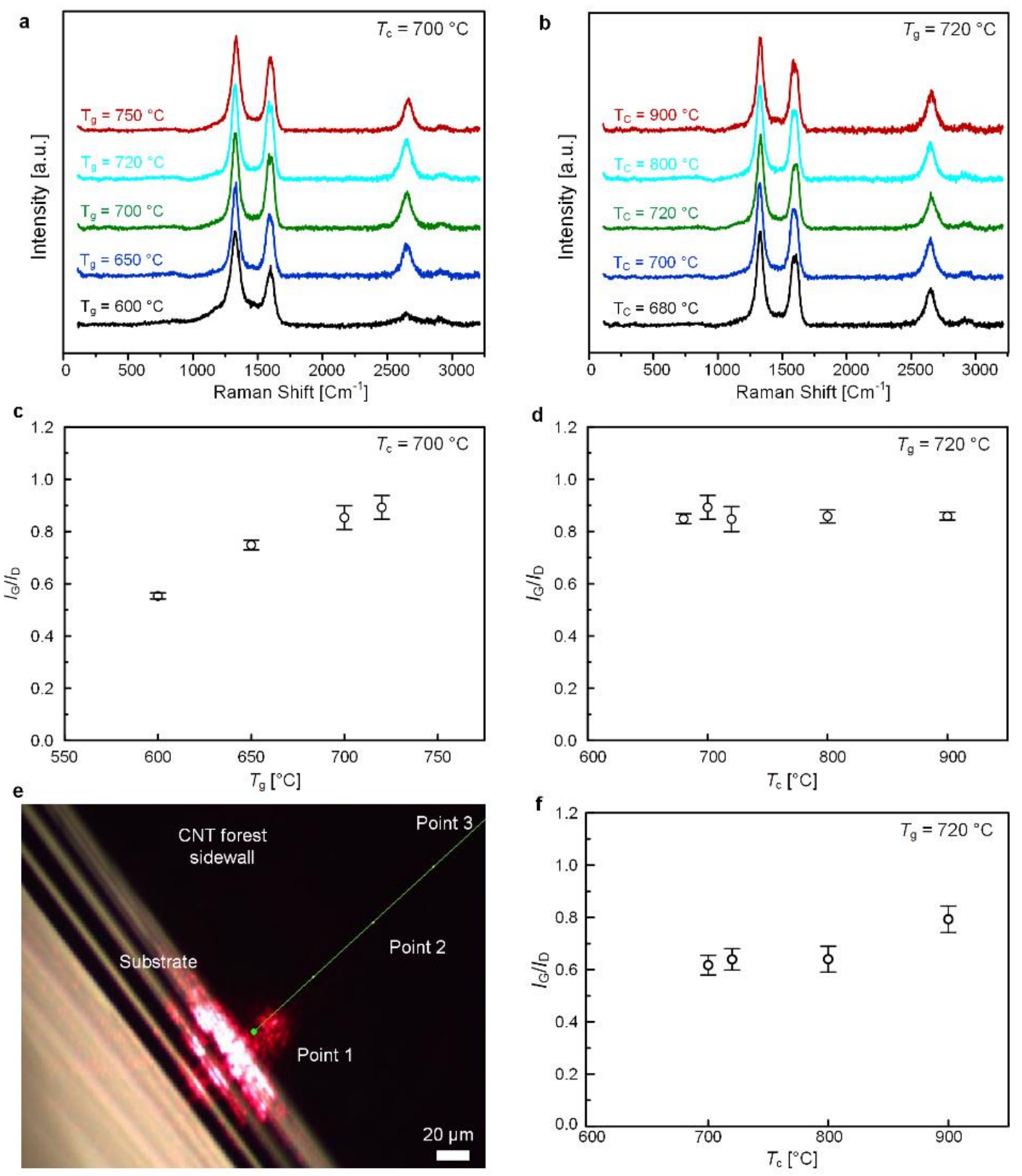

Fig. S3. Effect of $T_{\mathrm{c}}$ and $T_{\mathrm{g}}$ on structural quality of CNTs. (a) Representative Raman spectra obtained from the top of CNT forests grown at (a) $T_{\mathrm{c}}=700{ }^{\circ} \mathrm{C}$ and (b) $T_{\mathrm{g}}=720{ }^{\circ} \mathrm{C} . I_{\mathrm{G}} / I_{\mathrm{D}}$ ratios were obtained from top view Raman spectra of CNT forests (c) as a function of $T_{\mathrm{g}}$ at $T_{\mathrm{c}}=700{ }^{\circ} \mathrm{C}$ and (d) as a function of $T_{\mathrm{c}}$ at $T_{\mathrm{g}}=720^{\circ} \mathrm{C}$. (Error bars were obtained from multiple measurements from three forests in separate growth runs, and each forest was measured at three spots). (e) Depiction of how sidewall Raman spectra were obtained at three points, i.e., close to the substrate (point 1), in the middle (point 2), and close to the top of the forest (point3). (f) $I_{\mathrm{G}} / I_{\mathrm{D}}$ ratios obtained from sidewall Raman spectra of CNT forests as a function of $T_{\mathrm{c}}$ at $T_{\mathrm{g}}=720^{\circ} \mathrm{C}$. (Error bars were obtained from one forest measured at point 1,2, and 3 on the sidewall). The forest grown at $T_{\mathrm{c}}=$ $680^{\circ} \mathrm{C}$ was too short to obtain sidewall Raman spectrum. 

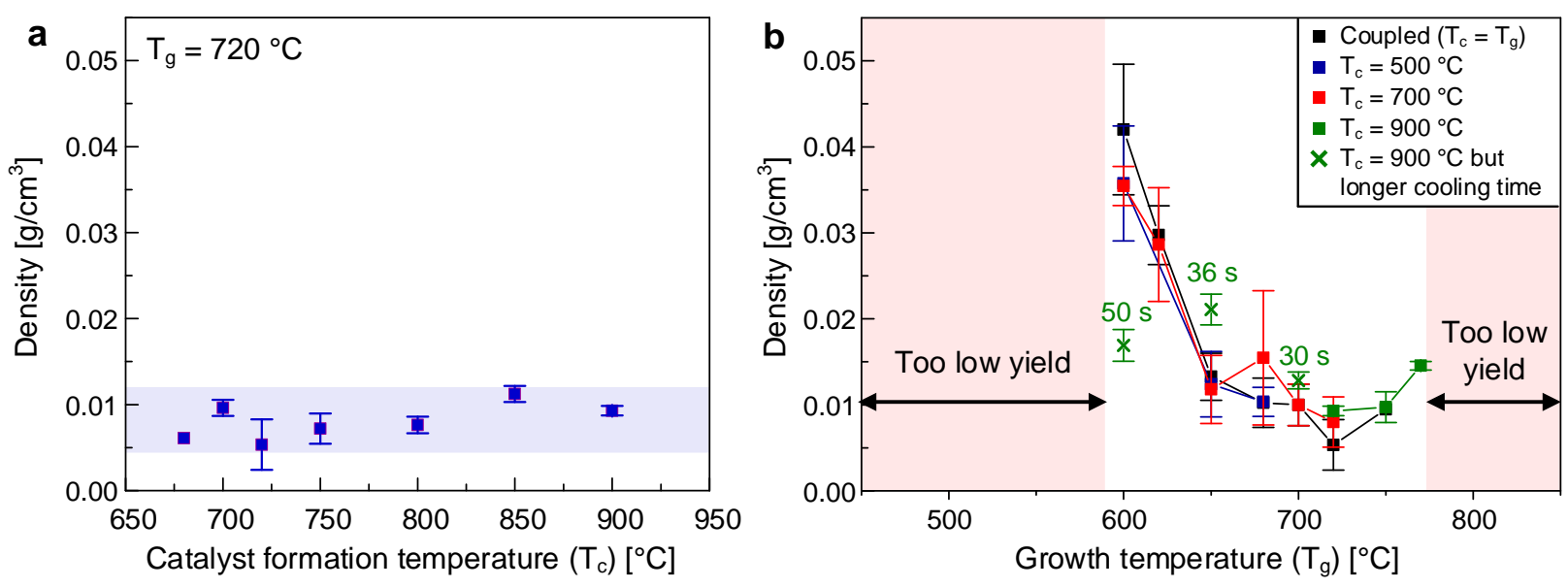

Fig. S4. Decoupled effects of $T_{c}$ and $T_{g}$ on CNT density of CNT forest: (a) density dependence on $T_{c}$ (for a constant $T_{g}=720^{\circ} \mathrm{C}$ ), and (b) density dependence on $T_{g}$ (for different $T_{c}$ ). At green point for $\mathrm{T}_{\mathrm{c}}=900{ }^{\circ} \mathrm{C}$, a longer period was needed for cooling down to $\mathrm{T}_{\mathrm{g}}$ below $700{ }^{\circ} \mathrm{C}$. The actual cooling time for those cases is noted next to the corresponding data points in (b). For the CNT forest density measurements (by the weight gain method), each CNT forest was grown until the growth was terminated by catalytic deactivation. We waited in each experiment for a sufficiently long growth time than the catalytic lifetime of each growth. The way we identified this in each case, was based on the real-time monitoring of growth kinetics. We monitored the growth in situ using a camera through the camera view port of our reactor, and ceased the supply of carbon source after the growth had been terminated (i.e. when forest height stops increasing in the videography). 


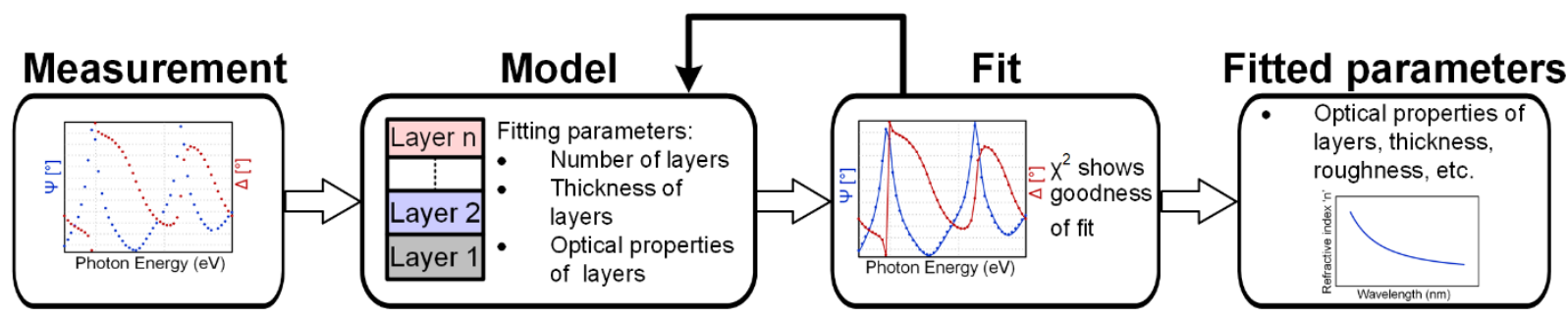

Fig. S5. Schematic representation of the multiple steps involved in ellipsometry measurements and data modeling. 

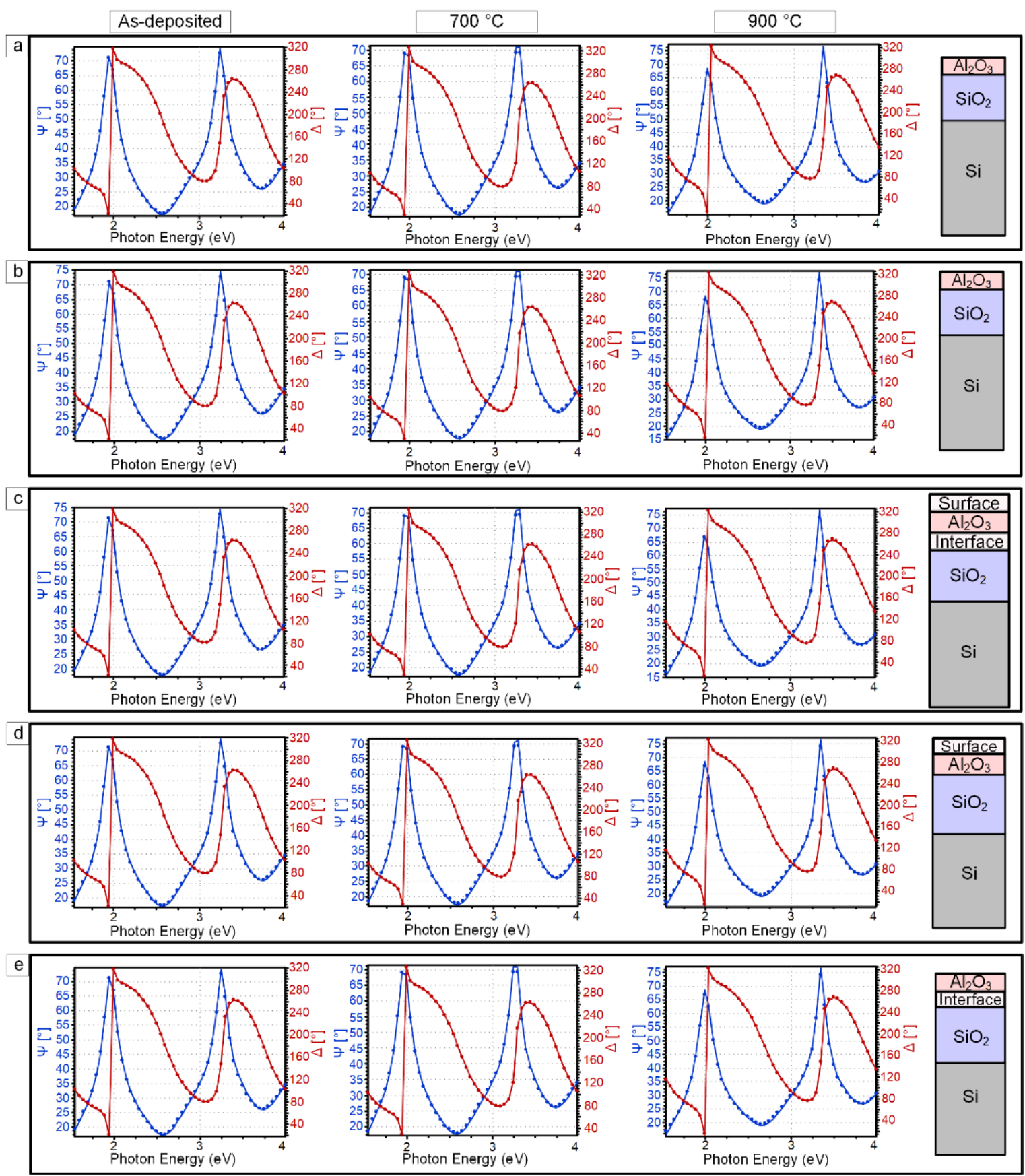

Fig. S6. Goodness of fit compared to experimental ellipsometry data (angles $\Psi$ and $\Delta$ ) for asdeposited alumina as well as alumina thermally treated at $700{ }^{\circ} \mathrm{C}$ and $900{ }^{\circ} \mathrm{C}$. The fits were obtained by using (a) $\mathrm{Al}_{2} \mathrm{O}_{3} / \mathrm{SiO}_{2} / \mathrm{Si}$ as the optical model and Sellmeier dispersion formula for alumina, (b) $\mathrm{Al}_{2} \mathrm{O}_{3} / \mathrm{SiO}_{2} / \mathrm{Si}$ as the optical model and Cauchy dispersion formula for alumina, (c) Surface layer $/ \mathrm{Al}_{2} \mathrm{O}_{3} / \mathrm{Al}_{2} \mathrm{O}_{3}-\mathrm{SiO}_{2}$ interface $/ \mathrm{SiO}_{2} / \mathrm{Si}$ as the optical model and Sellmeier dispersion formula for alumina, (d) Surface layer/ $/ \mathrm{Al}_{2} \mathrm{O}_{3} / \mathrm{SiO}_{2} / \mathrm{Si}$ as the optical model and Sellmeier dispersion formula for alumina, and (e) $\mathrm{Al}_{2} \mathrm{O}_{3} / \mathrm{Al}_{2} \mathrm{O}_{3}-\mathrm{SiO}_{2}$ interface $/ \mathrm{SiO}_{2} / \mathrm{Si}$ as the optical model and Sellmeier dispersion formula for alumina. The fit parameters of are listed in Tables S3-S7. 

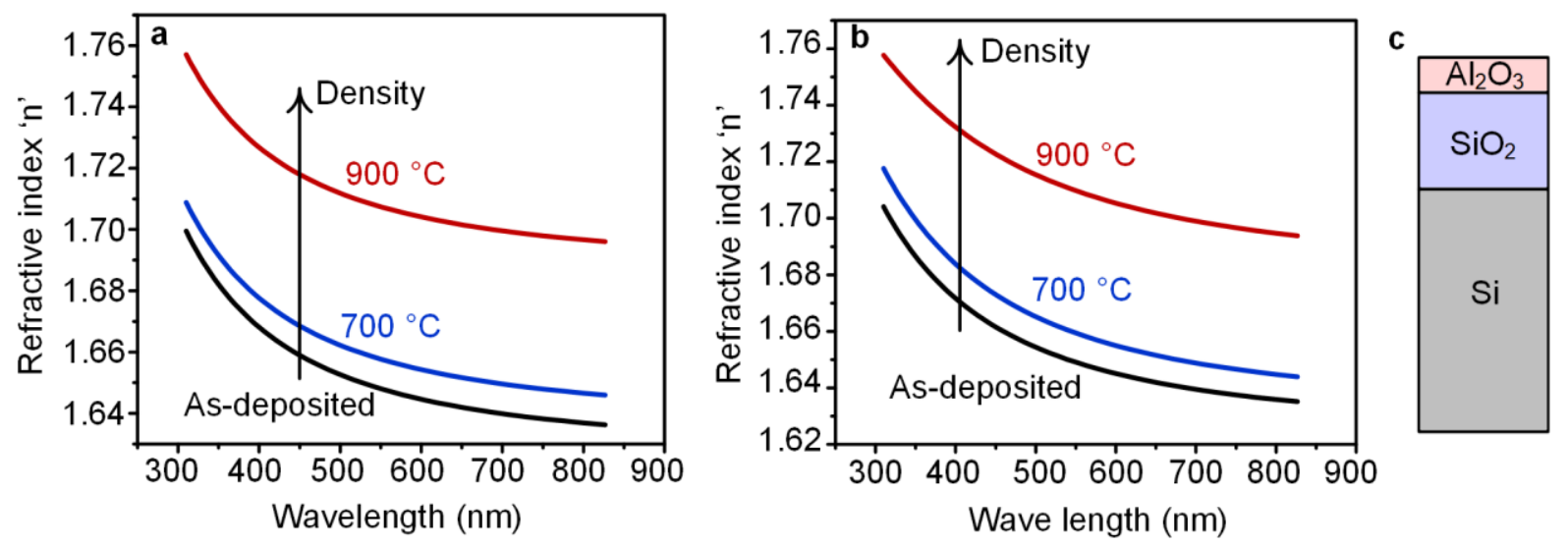

Fig. S7. Dispersion of the refractive index obtained by fitting ellipsometry data with (a) Sellmeier and (b) Cauchy functions based on (c) a simple model of the system $\left(\mathrm{Si} / \mathrm{SiO}_{2} / \mathrm{Al}_{2} \mathrm{O}_{3}\right)$. 

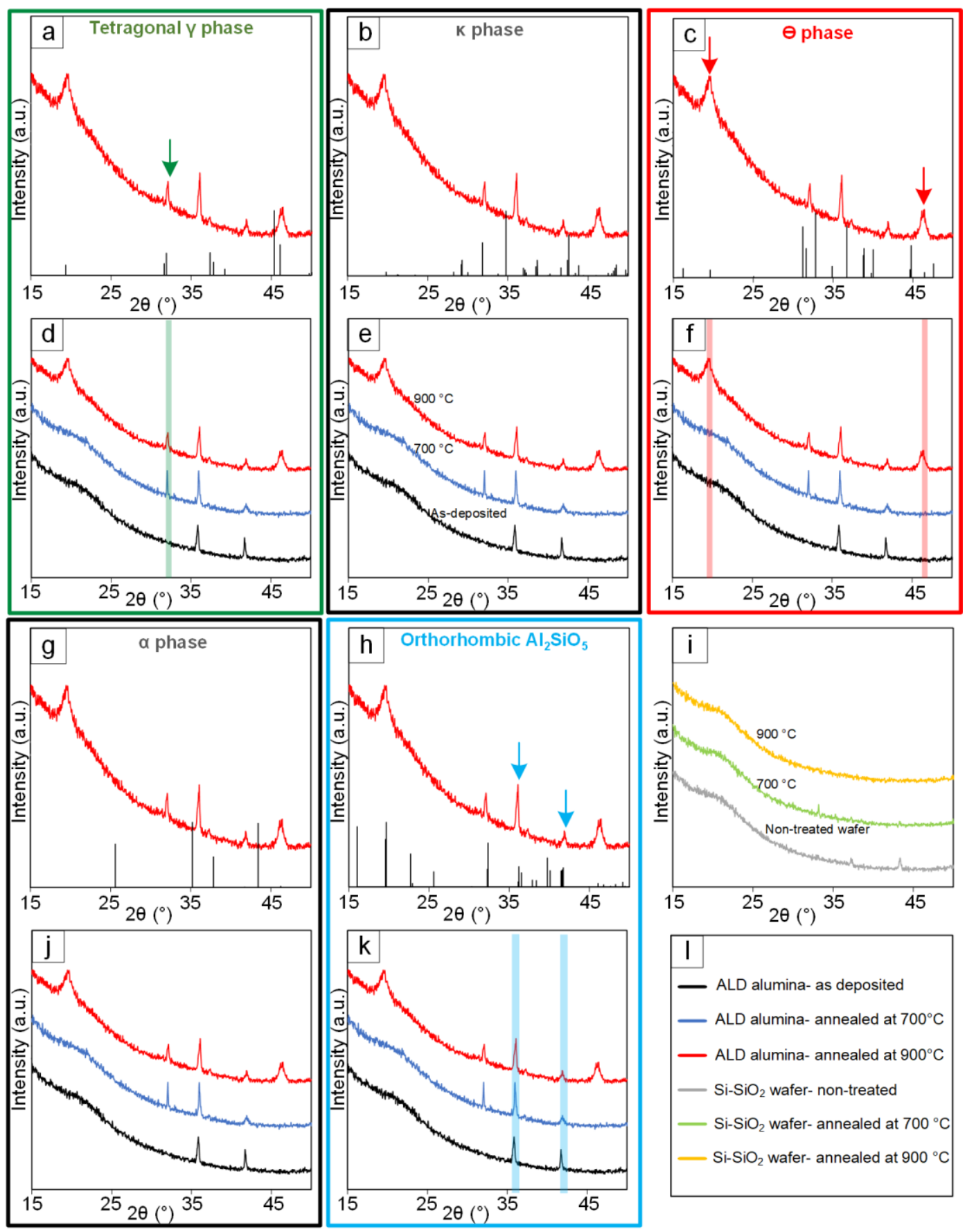

Fig. S8. Fitting XRD spectrum of the sample annealed at $900{ }^{\circ} \mathrm{C}$ with various alumina phases from ICSD: (a,d) tetragonal $\gamma$ phase [ICSD: 01-074-4629], (b,e) $\kappa$ phase [ICSD: 01-088-0107], (c,f) $\theta$ phase, $(\mathrm{g}, \mathrm{j}) \alpha$ phase [ICSD: 01-075-0783]. $(\mathrm{h}, \mathrm{k})$ shows the fitting of common peaks in all samples with the spectra of an orthorhombic aluminum silicon oxide. (i) shows the XRD spectrum for nontreated and annealed bare silicon wafer. 


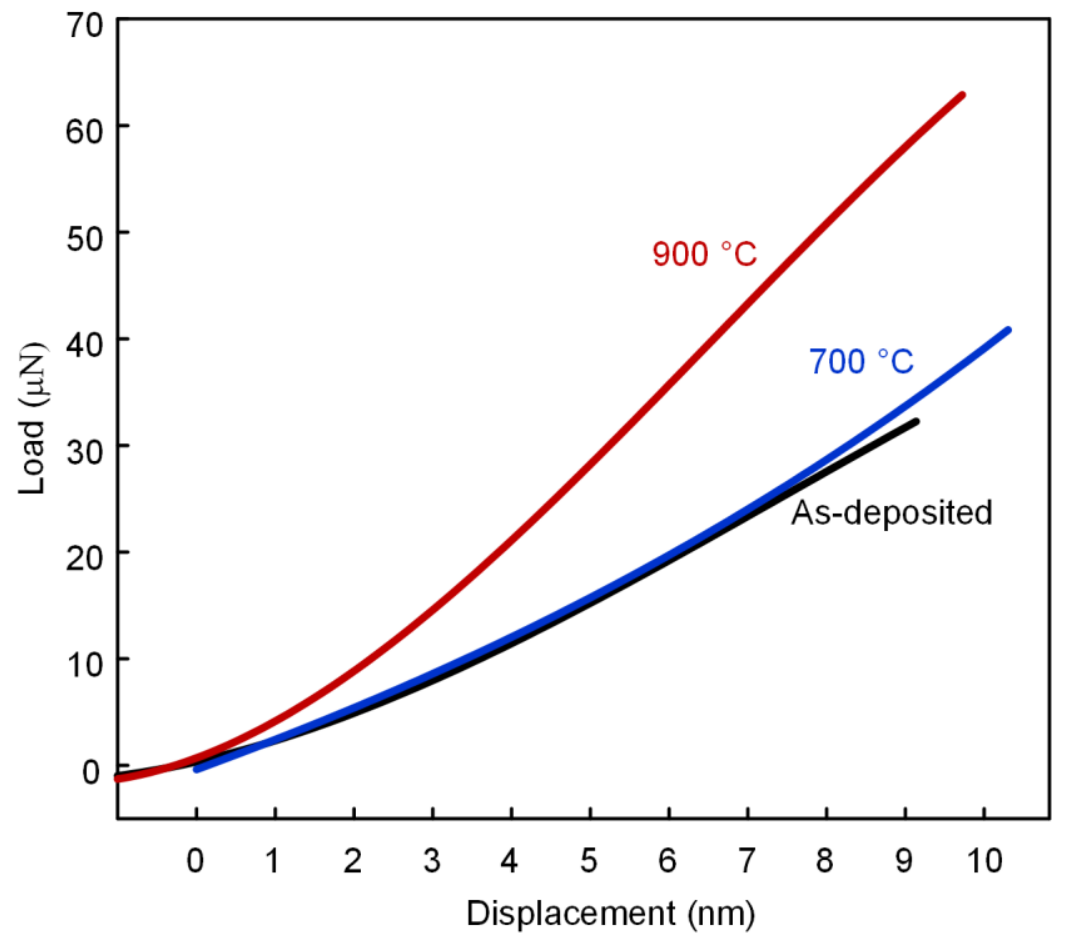

Fig. S9. Force-displacement curves of alumina films during loading stage of nanoindentation. 


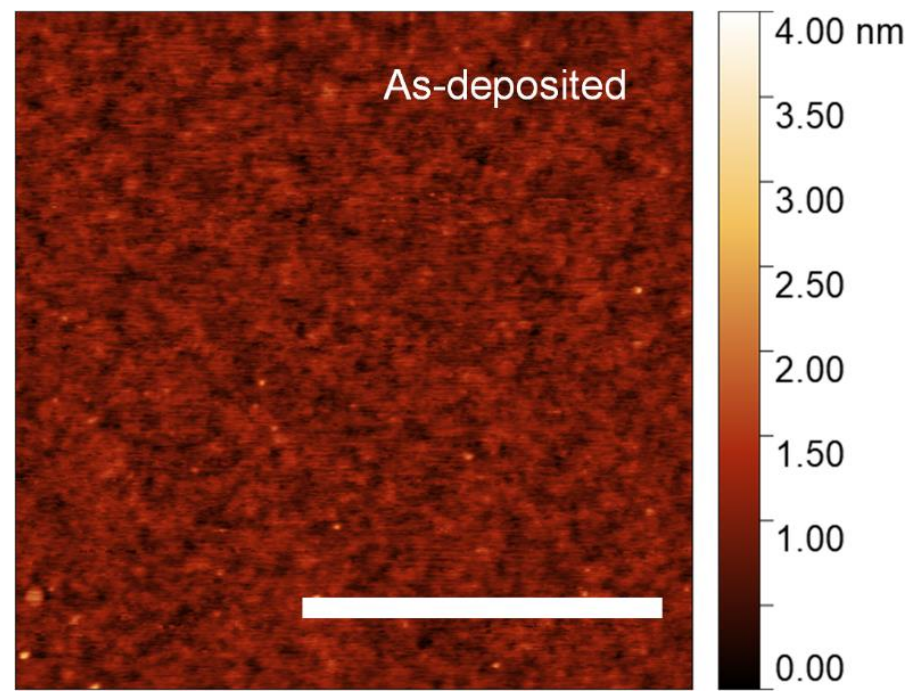

Fig. S10. AFM image of surface of as-deposited alumina layer. The scale bar is $500 \mathrm{~nm}$. 

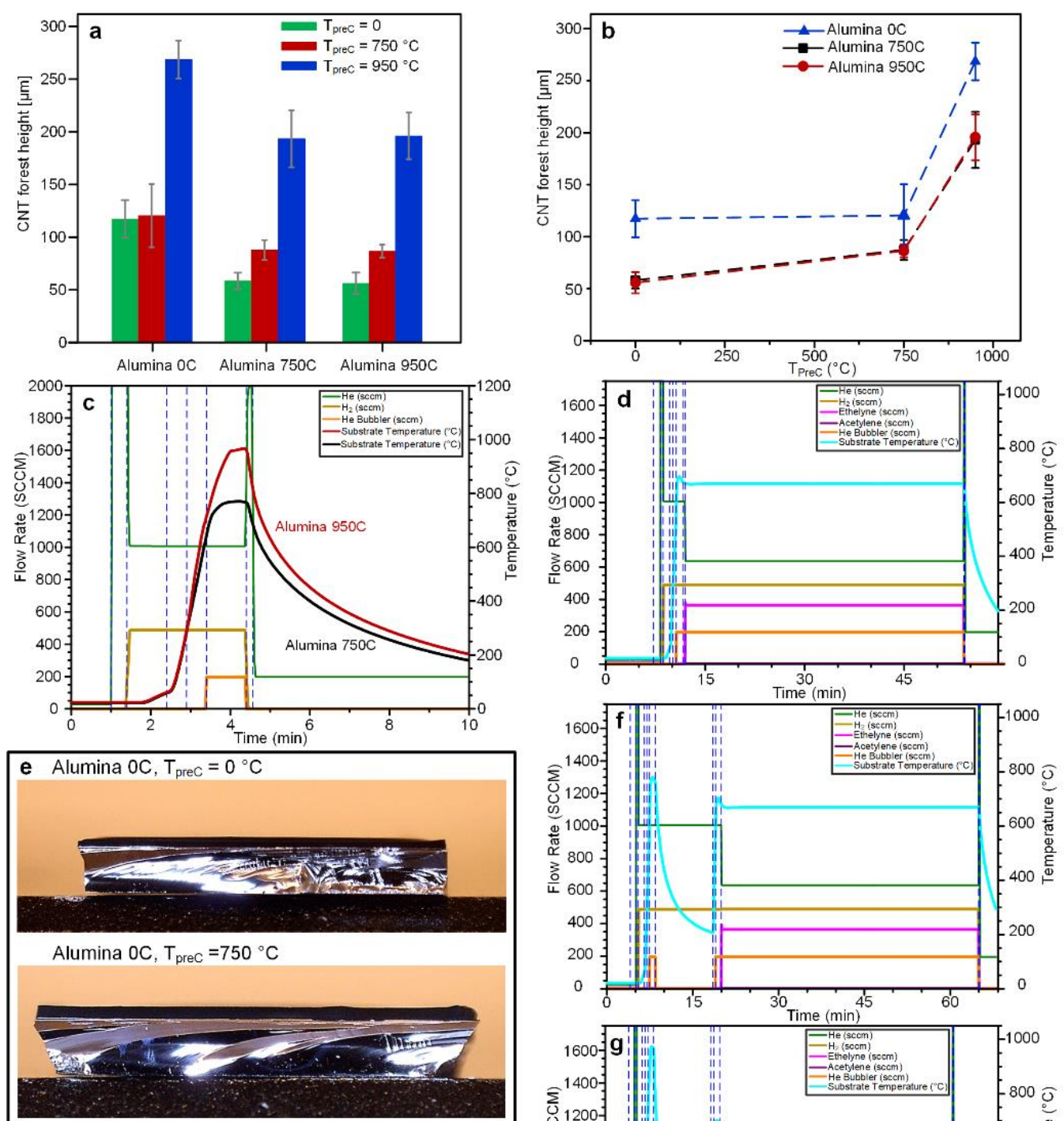

Alumina $0 \mathrm{C}, \mathrm{T}_{\text {prec }}=950^{\circ} \mathrm{C}$
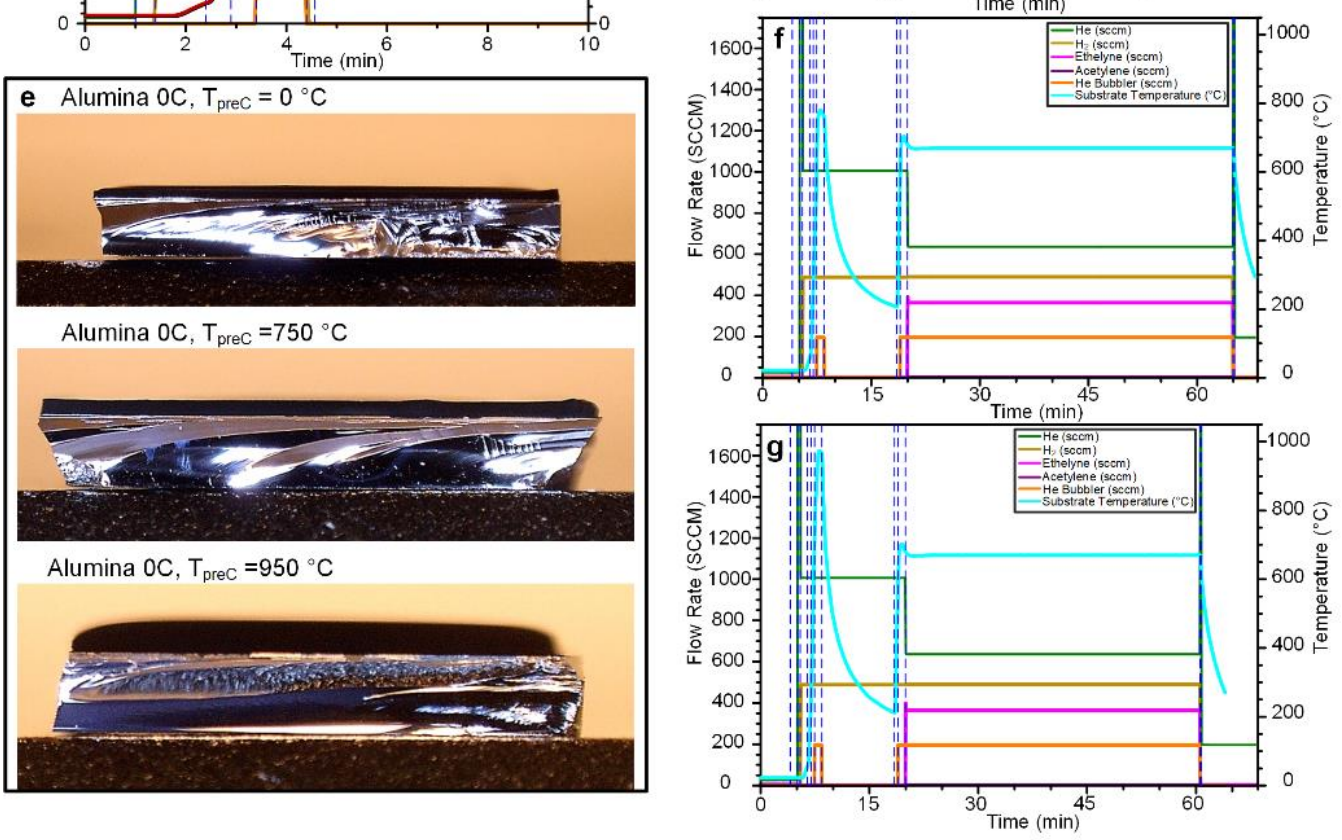

Fig. S11. (a) and (b) show that annealing alumina at $750{ }^{\circ} \mathrm{C}$ (Alumina 750C) prior to Fe deposition and annealing alumina at $950^{\circ} \mathrm{C}$ prior to Fe deposition (Alumina 950C) led to shorter forests compared to the case of no annealing prior to Fe deposition (Alumina 0C) in all growth recipes. Also, adding a catalyst pretreatment step at $T_{\text {preC }}=750{ }^{\circ} \mathrm{C}$ and $T_{\text {preC }}=950{ }^{\circ} \mathrm{C}$, where $T_{\text {pre }}$ represents the temperature of this additional pretreatment step, prior to the coupled growth recipe enhanced forest growth for all three types of samples. Error bars were obtained from multiple height measurements from all sides on one forest. (c) Annealing recipes used to prepare Alumina 750C and Alumina 950C samples. Growth recipes (d, f, g) all include a coupled recipe (at $T_{c}=T_{g}$ $=670{ }^{\circ} \mathrm{C}$ ). The recipes in (f) and (g) have an additional 1-minute pretreatment of the catalyst at 
$T_{\text {preC }}=750^{\circ} \mathrm{C}$ and $T_{\text {preC }}=950{ }^{\circ} \mathrm{C}$, respectively. (e) Optical images of the forests grown on Alumina OC samples with recipes ( $\mathrm{d}, \mathrm{f}$, and g) indicate that a pretreatment of the catalyst at $T_{\text {preC }}=950{ }^{\circ} \mathrm{C}$ significantly enhanced the growth. 
Table S1. Statistics of outer diameter and number of walls of CNTs grown at different $T_{\mathrm{c}}$ and $T_{\mathrm{g}}$ fitted by lognormal distribution.

\begin{tabular}{|l|l|l|l|l|}
\hline$T_{\mathrm{c}}$ and $T_{\mathrm{g}}$ & $\begin{array}{l}\text { Mean of outer } \\
\text { diameter [nm] }\end{array}$ & $\begin{array}{l}\text { standard } \\
\text { deviation of } \\
\text { outer } \\
\text { diameter [nm] }\end{array}$ & $\begin{array}{l}\text { Mode of outer } \\
\text { diameter [nm] }\end{array}$ & $\begin{array}{l}\text { Mode of } \\
\text { number of } \\
\text { walls }\end{array}$ \\
\hline$T_{\mathrm{c}}=700^{\circ} \mathrm{C}, T_{\mathrm{g}}=600{ }^{\circ} \mathrm{C}$ & 8.41 & 1.80 & 7.86 & 4 \\
\hline$T_{\mathrm{c}}=700^{\circ} \mathrm{C}, T_{\mathrm{g}}=700{ }^{\circ} \mathrm{C}$ & 8.35 & 1.67 & 7.88 & 3 \\
\hline$T_{\mathrm{c}}=900^{\circ} \mathrm{C}, T_{\mathrm{g}}=700{ }^{\circ} \mathrm{C}$ & 9.69 & 2.07 & 9.06 & 4 \\
\hline
\end{tabular}


Table S2. Statistics of height of nanoparticles formed by mock growths at different $T_{\mathrm{c}}$ and mock growth time fitted by lognormal distribution.

\begin{tabular}{|l|l|l|l|l|}
\hline $\mathrm{T}_{\mathrm{c}}$ and mock growth time & mean & standard & mode & skewness \\
\hline $700^{\circ} \mathrm{C}, 0$ minute & 8.22 & 2.36 & 7.30 & \\
\hline $700^{\circ} \mathrm{C}, 20$ minutes & 7.66 & 3.60 & 5.67 & 1.52 \\
\hline $700^{\circ} \mathrm{C}, 40$ minutes & 6.38 & 2.37 & 5.26 & 1.16 \\
\hline $700^{\circ} \mathrm{C}, 3$ hours & 4.98 & 1.79 & 4.15 & 1.13 \\
\hline $900^{\circ} \mathrm{C}, 0$ minute & 8.89 & 2.45 & 7.97 & 0.846 \\
\hline $900^{\circ} \mathrm{C}, 20$ minutes & 10.94 & 4.31 & 8.81 & 1.24 \\
\hline $900^{\circ} \mathrm{C}, 40$ minutes & 6.79 & 2.60 & 5.53 & 1.21 \\
\hline $900^{\circ} \mathrm{C}, 3$ hours & 6.00 & 2.25 & 4.93 & 1.17 \\
\hline
\end{tabular}

The distribution of height of nanoparticles was fitted by lognormal distribution, using the equation:

$$
\mathrm{y}=y_{0}+\frac{A}{w x \sqrt{2 \pi}} \exp \left(-\frac{(\ln x-\mu)^{2}}{2 w^{2}}\right)
$$

where $\mu$ and $w$ are the mean and standard deviation of the variable's natural logarithm, respectively.

Statistics were obtained using the following equations:

$$
\begin{gathered}
\text { mean }=\exp \left(\mu+\frac{w^{2}}{2}\right) \\
\text { standard deviation }=\exp \left(\mu+\frac{w^{2}}{2}\right) \sqrt{\exp \left(w^{2}\right)-1} \\
\text { mode }=\exp \left(\mu-w^{2}\right) \\
\text { skewness }=\exp \left(w^{2}+2\right) \sqrt{\exp \left(w^{2}\right)-1}
\end{gathered}
$$


Table S3. Fitted parameters using $\mathrm{Al}_{2} \mathrm{O}_{3} / \mathrm{SiO}_{2} / \mathrm{Si}$ as the optical model and Sellmeier dispersion formula.

\begin{tabular}{|c|c|c|c|c|c|}
\hline Optical model & Treatment & $\chi^{2}$ & $\mathrm{SiO}_{2}$ thickness (nm) & $\mathrm{Al}_{2} \mathrm{O}_{3}$ thickness (nm) & Sellmeier A \\
\hline $\mathrm{Al}_{2} \mathrm{O}_{3}$ & As deposited & 1.02 & $297.53 \pm 0.68$ & $103.12 \pm 0.55$ & $1.33 \pm 0.01$ \\
\hline \multirow{2}{*}{$\mathrm{Si}$} & Annealed at $700{ }^{\circ} \mathrm{C}$ & 1.54 & $297.80 \pm 0.80$ & $100.42 \pm 0.66$ & $1.36 \pm 0.01$ \\
\hline & Annealed at $900{ }^{\circ} \mathrm{C}$ & 2.45 & $301.83 \pm 0.77$ & $83.55 \pm 0.71$ & $1.53 \pm 0.01$ \\
\hline
\end{tabular}


Table S4. Fitted parameters using $\mathrm{Al}_{2} \mathrm{O}_{3} / \mathrm{SiO}_{2} / \mathrm{Si}$ as the optical model and Cauchy dispersion formula.

\begin{tabular}{|c|c|c|c|c|c|c|c|}
\hline \multirow{2}{*}{$\begin{array}{l}\text { Optical } \\
\text { model }\end{array}$} & \multirow[t]{2}{*}{ Treatment } & \multirow[t]{2}{*}{$\chi^{2}$} & \multirow{2}{*}{$\begin{array}{l}\mathrm{SiO}_{2} \\
\text { thickness } \\
(\mathrm{nm})\end{array}$} & \multirow{2}{*}{$\begin{array}{l}\mathrm{Al}_{2} \mathrm{O}_{3} \\
\text { thickness } \\
(\mathrm{nm})\end{array}$} & \multicolumn{3}{|c|}{ Cauchy parameters } \\
\hline & & & & & A & B & $\mathrm{C}$ \\
\hline \begin{tabular}{|l|}
$\mathrm{Al}_{2} \mathrm{O}_{3}$ \\
$\mathrm{SiO}_{2}$ \\
\end{tabular} & As deposited & 0.91 & $297.88 \pm 0.65$ & $\begin{array}{l}102.57 \pm \\
0.58\end{array}$ & $1.62 \pm 0.00$ & $0.75 \pm 0.20$ & $\begin{array}{l}0.02 \pm \\
0.16\end{array}$ \\
\hline \multirow{2}{*}{$\mathrm{Si}$} & $\begin{array}{l}\text { Annealed at } \\
700^{\circ} \mathrm{C}\end{array}$ & 1.15 & $298.34 \pm 0.70$ & $\begin{array}{l}99.50 \pm \\
0.63\end{array}$ & $1.63 \pm 0.01$ & $0.85 \pm 0.23$ & $\begin{array}{l}-0.02 \pm \\
0.18\end{array}$ \\
\hline & $\begin{array}{l}\text { Annealed at } \\
900^{\circ} \mathrm{C}\end{array}$ & 2.40 & $301.59 \pm 0.81$ & $\begin{array}{l}83.54 \pm \\
0.74\end{array}$ & $1.68 \pm 0.01$ & $0.97 \pm 0.31$ & $\begin{array}{l}-0.21 \pm \\
0.24\end{array}$ \\
\hline
\end{tabular}


Table S5. Fitted parameters using Surface layer/ $/ \mathrm{Al}_{2} \mathrm{O}_{3} / \mathrm{Al}_{2} \mathrm{O}_{3}-\mathrm{SiO}_{2}$ interface $/ \mathrm{SiO}_{2} / \mathrm{Si}$ as the optical model and Sellmeier dispersion formula.

\begin{tabular}{|c|c|c|c|c|c|c|c|}
\hline $\begin{array}{l}\text { Optical } \\
\text { model }\end{array}$ & Treatment & $\chi^{2}$ & $\begin{array}{l}\mathrm{SiO}_{2} \\
\text { thickness } \\
\text { (nm) }\end{array}$ & $\begin{array}{l}\text { Interface } \\
\text { roughness } \\
(\mathrm{nm})\end{array}$ & $\begin{array}{l}\mathrm{Al}_{2} \mathrm{O}_{3} \\
\text { thickness } \\
(\mathrm{nm})\end{array}$ & $\begin{array}{l}\text { Surface } \\
\text { roughness } \\
(\mathrm{nm})\end{array}$ & $\begin{array}{l}\text { Sellmeier } \\
\text { A }\end{array}$ \\
\hline \begin{tabular}{|c|} 
Surface \\
$\mathrm{Al}_{2} \mathrm{O}_{3}$ \\
Interface \\
\end{tabular} & $\begin{array}{l}\text { As } \\
\text { deposited }\end{array}$ & 0.72 & $\begin{array}{l}288.60 \pm \\
2.22\end{array}$ & $19.59 \pm 4.51$ & $\begin{array}{l}91.10 \pm \\
2.97\end{array}$ & $2.34 \pm 0.71$ & $\begin{array}{l}1.33 \pm \\
0.01\end{array}$ \\
\hline $\mathrm{SiO}_{2}$ & $\begin{array}{l}\text { Annealed at } \\
700^{\circ} \mathrm{C}\end{array}$ & 0.87 & $\begin{array}{l}287.11 \pm \\
2.00\end{array}$ & $24.98 \pm 4.25$ & $\begin{array}{l}83.79 \pm \\
3.17\end{array}$ & $3.95 \pm 0.88$ & $\begin{array}{l}1.37 \pm \\
0.01\end{array}$ \\
\hline $\mathrm{Si}$ & $\begin{array}{l}\text { Annealed at } \\
900^{\circ} \mathrm{C}\end{array}$ & 2.32 & $\begin{array}{l}287.52 \pm \\
2.33\end{array}$ & $28.58 \pm 4.96$ & $\begin{array}{l}66.84 \pm \\
4.16\end{array}$ & $1.71 \pm 1.27$ & $\begin{array}{l}1.59 \pm \\
0.03\end{array}$ \\
\hline
\end{tabular}


Table S6. Fitted parameters using Surface layer $/ \mathrm{Al}_{2} \mathrm{O}_{3} / \mathrm{SiO}_{2} / \mathrm{Si}$ as the optical model and Sellmeier dispersion formula.

\begin{tabular}{|c|c|c|c|c|c|c|}
\hline $\begin{array}{l}\text { Optical } \\
\text { model }\end{array}$ & Treatment & $\chi^{2}$ & $\begin{array}{l}\mathrm{SiO}_{2} \text { thickness } \\
(\mathrm{nm})\end{array}$ & $\begin{array}{l}\mathrm{Al}_{2} \mathrm{O}_{3} \text { thickness } \\
(\mathrm{nm})\end{array}$ & $\begin{array}{l}\text { Surface roughness } \\
(\mathrm{nm})\end{array}$ & $\begin{array}{l}\text { Sellmeier } \\
\text { A }\end{array}$ \\
\hline \begin{tabular}{|c|} 
Surface \\
$\mathrm{Al}_{2} \mathrm{O}_{3}$ \\
\end{tabular} & As deposited & 0.82 & $298.39 \pm 0.68$ & $101.83 \pm 0.66$ & $1.53 \pm 0.47$ & $\begin{array}{l}1.32 \pm \\
0.01\end{array}$ \\
\hline $\mathrm{SiO}_{2}$ & $\begin{array}{l}\text { Annealed at } \\
700{ }^{\circ} \mathrm{C}\end{array}$ & 1.05 & $299.31 \pm 0.78$ & $98.26 \pm 0.80$ & $2.35 \pm 0.59$ & $\begin{array}{l}1.35 \pm \\
0.01\end{array}$ \\
\hline $\mathrm{Si}$ & $\begin{array}{l}\text { Annealed at } \\
900{ }^{\circ} \mathrm{C}\end{array}$ & 2.48 & $301.87 \pm 0.77$ & $83.52 \pm 0.72$ & $0.01 \pm 0.20$ & $\begin{array}{l}1.53 \pm \\
0.01\end{array}$ \\
\hline
\end{tabular}


Table S7. Fitted parameters using $\mathrm{Al}_{2} \mathrm{O}_{3} / \mathrm{Al}_{2} \mathrm{O}_{3}-\mathrm{SiO}_{2}$ interface/SiO $2 / \mathrm{Si}$ as the optical model and Sellmeier dispersion formula.

\begin{tabular}{|c|c|c|c|c|c|c|}
\hline $\begin{array}{l}\text { Optical } \\
\text { model }\end{array}$ & Treatment & $\chi^{2}$ & $\begin{array}{l}\mathrm{SiO}_{2} \text { thickness } \\
(\mathrm{nm})\end{array}$ & $\begin{array}{l}\text { Interface roughness } \\
(\mathrm{nm})\end{array}$ & $\begin{array}{l}\mathrm{Al}_{2} \mathrm{O}_{3} \text { thickness } \\
(\mathrm{nm})\end{array}$ & $\begin{array}{l}\text { Sellmeier } \\
\text { A }\end{array}$ \\
\hline \begin{tabular}{|c|}
$\mathrm{Al}_{2} \mathrm{O}_{3}$ \\
Interface \\
\end{tabular} & As deposited & 0.98 & $293.34 \pm 3.48$ & $8.09 \pm 6.54$ & $99.13 \pm 3.35$ & $\begin{array}{l}1.33 \pm \\
0.01\end{array}$ \\
\hline $\mathrm{SiO}_{2}$ & $\begin{array}{l}\text { Annealed at } \\
700{ }^{\circ} \mathrm{C}\end{array}$ & 1.52 & $294.38 \pm 3.82$ & $6.56 \pm 7.04$ & $97.20 \pm 3.57$ & $\begin{array}{l}1.36 \pm \\
0.01\end{array}$ \\
\hline נו & $\begin{array}{l}\text { Annealed at } \\
900^{\circ} \mathrm{C}\end{array}$ & 2.48 & $301.71 \pm 0.82$ & $0.29 \pm 0.49$ & $83.39 \pm 0.75$ & $\begin{array}{l}1.53 \pm \\
0.01\end{array}$ \\
\hline
\end{tabular}

